\title{
Comparison of Morphological Changes of Muscle Fibers in Response to Dynamic Electrical Muscle Contraction and Dynamic Hydraulic Stimulation in a Rat Hindlimb Disuse Model
}

\author{
M. HU ${ }^{1}$, H. LAM ${ }^{1}$, R. YEH ${ }^{1}$, M. TEERATANANON ${ }^{1}$, Y.-X. QIN ${ }^{1}$ \\ ${ }^{1}$ Department of Biomedical Engineering, Stony Brook University, Stony Brook, NY, USA
}

Received June 12, 2016

Accepted September 2, 2016

On-line February 28, 2017

\begin{abstract}
Summary
This study attempted to compare the muscle fiber morphological responses to dynamic electrical muscle stimulation (DEMS) and dynamic hydraulic stimulation (DHS) in rats under hindlimb suspension (HLS). DEMS at $1 \mathrm{~Hz}, 50 \mathrm{~Hz}$ and $100 \mathrm{~Hz}$ for $10 \mathrm{~min} /$ day, 5 days/week were introduced to the animals' right quadriceps. Static and $2 \mathrm{~Hz}$ DHS were introduced to the right tibiae of other animal groups on a $10 \mathrm{~min}$ on $-5 \mathrm{~min}$ off -10 min on" loading regime for 5 days/week. In the end of the 4-week experiments, histological changes in the corresponding soleus, gastrocnemius and quadriceps of the stimulated sites were examined. Compared to age-matched, HLS led to muscle atrophy and strongly reduced muscle wet weights and averaged cross-sectional fiber areas. Among the tested DEMS frequencies, the averaged cross-sectional quadriceps fiber area in the $50 \mathrm{~Hz}$ group was $29 \%$ larger than the $100 \mathrm{~Hz}$ group. In contrast, difference in the muscle fiber response to the static and $2 \mathrm{~Hz}$ DHS was not observed in either soleus or gastrocnemius. Muscle fiber morphological responses to the active DEMS was in a load frequency dependent manner under disuse condition. Relatively passive compressions, either via static or $2 \mathrm{~Hz}$ DHS, were unable to induce any difference in the muscle fiber responses under functional disuse.
\end{abstract}

\section{Key words}

Disuse muscle atrophy - Hindlimb unloading • Mechanical stimulation • Muscle fiber regeneration

\section{Corresponding author}

Y.-X. Qin, Department of Biomedical Engineering, Stony Brook University, Bioengineering Bldg., Rm 215, Stony
Brook, NY 11794-5281, USA. Fax: 631-632-8577. E-mail: Yi-Xian.Qin@stonybrook.edu

\section{Introduction}

Skeletal muscle and bone are closely interacted both anatomically and functionally (Cianferotti and Brandi 2014). Both of these tissues have been shown to be dramatically affected by the lack of gravity and immobilization (Bonewald et al. 2013). Biomechanical signals or the triggered systemic hormonal stimuli, elicited from conditions such as loading or disuse, are sensed and transduced by the endocrine properties of muscle and bone (Cianferotti and Brandi 2014). Despite the assumption that the major effect of a lack of gravity could be due to a lack of muscle loading on the bone, muscle and bone tissue may actually contribute to each other's restoration or repair (Bonewald et al. 2013). Therefore, a single duo-functional therapeutic that could maintain and heal not only bone but also muscle is most ideal and needed to be identified.

Decreases in muscle activity leading to muscle atrophy has been reported following space mission, spinal cord injury, bed rest and aging. The alteration in mechanical environment rapidly induces muscular adaptation in fiber size and metabolic properties (LeBlanc et al. 2000, Edgerton et al. 1995, Zhou et al. 1995, Gregory et al. 2003, Narici et al. 2003, Trappe et al. 2001). Short-term spaceflight caused significant losses in muscle volume by 5 to $17 \%$, fiber cross-sectional area by 11 to $24 \%$, and type I fiber by 6 to $8 \%$ (LeBlanc et al. 2000, Edgerton et al. 1995). Likewise, 6-week post spinal 
cord injury caused a $15 \%$ lean muscle mass loss at the lower extremities and an averaged muscle cross-sectional areas reduction by 18 to $46 \%$ (Castro et al. 1999, Spungen et al. 2003). These changes are often associated with increased muscle weakness and fracture risk (Zehnder et al. 2004).

Small animal models have been extensively studied to mimic the condition of disuse (Allen and Bloomfield 2003, Morey-Holton and Globus 2002, Hauschka et al. 1988, Morey-Holton and Globus 1998). Similar to human, rodent studies displayed a loss of skeletal muscle mass, greatest in the postural muscles, and decreased in muscle force (Boonyarom and Inui 2006, Fisher et al. 1998, Hurst and Fitts 2003, McClung et al. 2006). Chemical-induced paralyzed limb in rats showed the highest decline $(\sim 40 \%)$ in muscle weight and fiber cross-sectional area in the soleus muscle (Dupont Salter et al. 2003). The degree of muscle atrophy was not as profound in the tibialis anterior and gastrocnemius, $\sim 20 \%$. Rat hindlimb unloading also supported these findings, where there was significantly reduction in both type I and type II muscle fibers area for the soleus and plantaris muscles (Kyparos et al. 2005). In addition, hindlimb suspension decreased the percentage of type I muscle fibers by an approximately $40 \%$, while the number of type II fibers were augmented by $\sim 20 \%$ (Kyparos et al. 2005).

As an intervention, functional electrical stimulation produces isometric contractions in replacement for the lack of muscle activity (Baldi et al. 1998). The induced contractions have been shown to increase muscle volume, muscle cross-sectional area, and improve fatigue resistance in human (Gerrits et al. 2002, Skold et al. 2002). However, the effects of this external stimulus highly depended on the variability in the duration and frequency of the stimulation (Perez et al. 2002). Effect of dynamic electrical muscle stimulation (DEMS) on mitigation of disuse bone loss in rats has been shown in a frequency and contraction-to-rest ratio dependent manner (Lam and Qin 2008, Lam et al. 2011). On the other hand, the newly developed dynamic hydraulic stimulation (DHS) is suggested to act noninvasively as an external oscillatory muscle coupling. The dynamic components derived from the hydraulic loading have been shown to influence bone interstitial fluid pressure and mitigate disuse bone loss in a frequency and magnitude dependent manner ( $\mathrm{Hu}$ et al. 2014, Hu et al. 2013a, Hu et al. 2012), possibly through the modulation on the bone marrow mesenchymal stem cell population ( $\mathrm{Hu}$ et al. 2013b) and osteogenic gene expressions (Hu and Qin 2014).

Electrical stimulation applied to animals has reported various effects on the skeletal muscle morphology and biochemistry. Numerous stimulation paradigms has been tested and generated mixed conclusion on the benefit of such stimulations. The discrepancies were mainly contributed by the applied stimulation frequency, the duration of the stimulation, and the type of muscle analyzed (Mabuchi et al. 1982, Kernell et al. 1987, Gorza et al. 1988, Jarvis et al. 1996). Stimulation at $10 \mathrm{~Hz}$ for $24 \mathrm{~h}$ per day increased fatigue resistance yet decreased the size of the tibialis anterior muscles (Salmons and Henriksson 1981). Lower frequency, such as $2 \mathrm{~Hz}$, applied for $10 \mathrm{~h}$ per day demonstrated a marked reduction in paralyzed soleus atrophy (Dupont Salter et al. 2003). Most studies that showed positive effects of electrical muscle contraction at the tissue level have used extremely long stimulation protocols, from 2 to $24 \mathrm{~h}$. In addition, the application of the stimulation frequency ranged from 1 to $100 \mathrm{~Hz}$.

As skeletal muscle and bone are interrelated and inter-dependent, and that optimized stimulation signals are crucial for tissue regeneration, we were interested in investigating the frequency effect of DEMS and the effect of the dynamic components derived from DHS on the muscle fiber morphological responses under disuse condition. Separate animal studies were performed: (1) to verify the atrophic effect of hindlimb suspension on rats' soleus, gastrocnemius, and quadriceps muscles; (2) to evaluate the difference in muscle fiber morphological responses to DEMS at $1 \mathrm{~Hz}, 50 \mathrm{~Hz}$ and $100 \mathrm{~Hz}$; and (3) to access the difference in muscle fiber morphological responses to static and $2 \mathrm{~Hz}$ DHS loading.

\section{Materials and Methods}

Animal study 1 - Validation of HLS induced muscle atrophy

The effect of lacking daily activity on muscle morphological change was investigated. Stony Brook University IACUC approved all the animal experimental procedures. Five-month-old female Sprague Dawley virgin rats (Charles River, MA) were randomly divided into three groups: (1) baseline, (2) age-matched and (3) HLS ( $n=5$ per group). The animals in the baseline group were sacrificed at the beginning of the study. The HLS procedure was similar to the setup from our previous studies (Hu et al. 2012). Briefly, the tail of each animal 
was cleaned with $70 \%$ alcohol and lightly coated with tincture of benzoin (Bruce Medial Supply, MA), while the animal was under standard isoflurane anesthesia. The tail harness was made from a paperclip-looped plastic tab with a strip of skin tape. Once the tail was dried, the skin tape was adhered to the tail, covering $3 / 4$ of its length. The tape was secured with two strips of filament tapes; one over the end of the skin tape around the base of the tail and the other about half-way up. The tail harness was then attached to a fish-line swivel apparatus that hangs from the top of the 18 "x18"x24" (LxWxH) stainless steel cage. An approximately $30^{\circ}$ head-down tilt was set to prevent contact of the animal's hindlimbs and the cage bottom. The animal's forelimbs were allowed full access to the entire cage bottom. Animals were provided with standard rodent chow and water ad libitum, and roomed under temperature-controlled and a 12:12 h light:dark cycle. Animals' body weights and overall health were carefully monitored throughout the 4-week HLS study. At the end of the study, the animals were euthanized by $\mathrm{CO}_{2}$; the soleus, gastrocnemius and quadriceps muscles were extracted. Measurements of the animals' end-point body weights normalized to the corresponded baseline, as well as the muscle wet weights of the right soleus, gastrocnemius and quadriceps were recorded. The samples were then snap-frozen using OCT compound (Tissue Tek, USA) and liquid $\mathrm{N}_{2}$-chilled 2-Methylbutane, and then stored at $-80^{\circ} \mathrm{C}$.

Animal study 2 - Effect of DEMS on muscle fiber morphology at various loading frequencies

The effect of DEMS on muscle fiber morphology at various loading frequencies under disuse condition was examined via a 4-week HLS study. Animals were randomly assigned to groups of: 1) HLS+1 Hz DEMS ( $n=7), 2) \mathrm{HLS}+50 \mathrm{~Hz}$ DEMS ( $\mathrm{n}=4)$ and 3) HLS+100 Hz DEMS ( $\mathrm{n}=4)$. Functional disuse was induced by HLS, using the same setup as described above. DEMS was applied in conjunction with HLS for 4 weeks. For the daily stimulation, DEMS was applied via two needle-size electrodes (L-type gauge \#3, Seirin, Weymouth, MA) to the isoflurane anesthetized animals. One electrode was placed at the right lateral proximal quadriceps (rectus femoris), and the other was placed at the lateral distal quadriceps (rectus femoris). The electrodes were then connected to a function generator (Model 395, Wavetek, USA) to transmit a $1 \mathrm{~ms}$ square pulse with various stimulation frequencies $(1 \mathrm{~Hz}, 50 \mathrm{~Hz}$ and $100 \mathrm{~Hz}$ ). The stimulation duration was 10 min per day for 5 days per week. To avoid muscle fatigue, a restinsertion period ( $2 \mathrm{~s}$ contraction followed by $8 \mathrm{~s}$ rest) was added in the DEMS regimen. The animals were euthanized at the end of the 4-week study via $\mathrm{CO}_{2}$. Muscle samples (right soleus, rectus femoris of the right quadriceps and lateralis of the right gastrocnemius) were harvested immediately after sacrifice and then cryo-preserved.

Animal study 3 - Effect of dynamic components of DHS on muscle fiber morphology

A 4-week HLS study was conducted to evaluate the effect of the dynamic components of DHS on muscle fiber morphology. Five-month-old female Sprague Dawley virgin rats (Charles River, MA) were randomly divided into two groups: 1) HLS+static loading ( $\mathrm{n}=5)$ and 2) $\mathrm{HLS}+\mathrm{DHS}$ at $2 \mathrm{~Hz} \quad(\mathrm{n}=6)$. Daily $20 \mathrm{~min}$ loading (10 min on -5 min off - $10 \mathrm{~min}$ on) for 5 days/week was applied in conjunction with HLS. To deliver DHS, a costume design inflatable cuff was placed over around the right hindlimb over the region of the tibia. An oscillatory actuator-driven syringe, a force-controlled syringe, and a pressure sensor were connected to the stimulation cuff to deliver dynamic compression with a constant static pressure by monitoring using the pressure sensor. A programmable waveform/signal generator (HP33120A, Hewlett-Packard, Palo Alto, CA) was used to control the actuator-driven syringe, which supported the inflation and deflation of the cuff. The DHS was applied to the stimulated animals at $30 \mathrm{~mm} \mathrm{Hg}$ static pressure $+30 \mathrm{~mm} \mathrm{Hg}(\mathrm{p}-\mathrm{p})$ dynamic pressure with $2 \mathrm{~Hz}$ of loading frequency. Animals in the static group only received $30 \mathrm{~mm} \mathrm{Hg}$ static pressure yet for the same amount of time. The rats were euthanized via $\mathrm{CO}_{2}$ at the end of the study, and the right soleus and lateralis of the gastrocnemius were obtained and cryo-preserved.

\section{Histology and image analysis}

Cross-sections of the cryo-preserved muscle samples, containing both deep and superficial muscle layers, were made to $8 \mu \mathrm{m}$ using a cryostat (Leica) at $-20^{\circ} \mathrm{C}$. Three sections were then stained with Hemotoxilyn and Eoson (H\&E, Polyscience, USA). Five random cross-sectional bright field images (20x) were captured from each muscle section with a Zeiss microscope (AxioVision 4.5, Germany). Averaged crosssectional muscle fiber areas were determined by Image $\mathbf{J}$ software and normalized to the averaged HLS value. 


\section{Statistical analysis}

The percent body weight change was reported as mean \pm SE. The values of the muscle wet weight and evaluations of the averaged cross-sectional muscle fiber area were reported as mean $\pm \mathrm{SD}$. For each analysis, significance differences between groups were determined using GraphPad Prism 3.0 Software (GraphPad Software InC., La Jolla, CA). One-way ANOVA with Tukey's pairwise multiple comparison tests and unpaired Student's T Test was performed, respectively to the analysis on multiple groups or two groups. The level of significance was considered at $p<0.05$.

\section{Results}

\section{HLS induced body weight change and muscle atrophy}

To validate the impact of HLS on the animals' skeletal muscles, the animals' body weights, muscle wet weights and averaged cross-sectional muscle fibers were evaluated. Age-matched animals showed an increase in body weight by $2.5 \%$ (Fig. 1). On the other hand, HLS reduced the body weight by $6.2 \% \quad(p<0.05$ vs. age-matched). Additionally, HLS significantly decreased the right soleus muscle wet weight compared to the baseline $(-54 \% ; p<0.001)$ and the age-matched $(-57 \%$; $p<0.001)$ groups, while the right soleus muscle wet weight of the age-matched group was similar to the baseline (Fig. 2). Similarly, the muscle wet weights of the right gastrocnemius were not significantly different between baseline and age-matched control. However, HLS significantly reduced the right gastrocnemius wet weight compared to the baseline $(-25 \% ; p<0.01)$ and age-matched $(-27 \% ; p<0.001)$ groups. HLS also significantly reduced the right quadriceps wet weight compared to the age-matched control $(-14 \% ; p<0.05)$. For the averaged cross-sectional muscle fiber areas (Fig. 3), HLS negatively impacted the right soleus (-56\% at $p<0.001$ vs. baseline; $-49 \%$ at $p<0.001$ vs. age-matched) and right quadriceps (-26\% at $p<0.05$ vs. age-matched) but not the right gastrocnemius ( $p>0.05$ vs. baseline or age-matched control).

\section{Change of Body Weight}

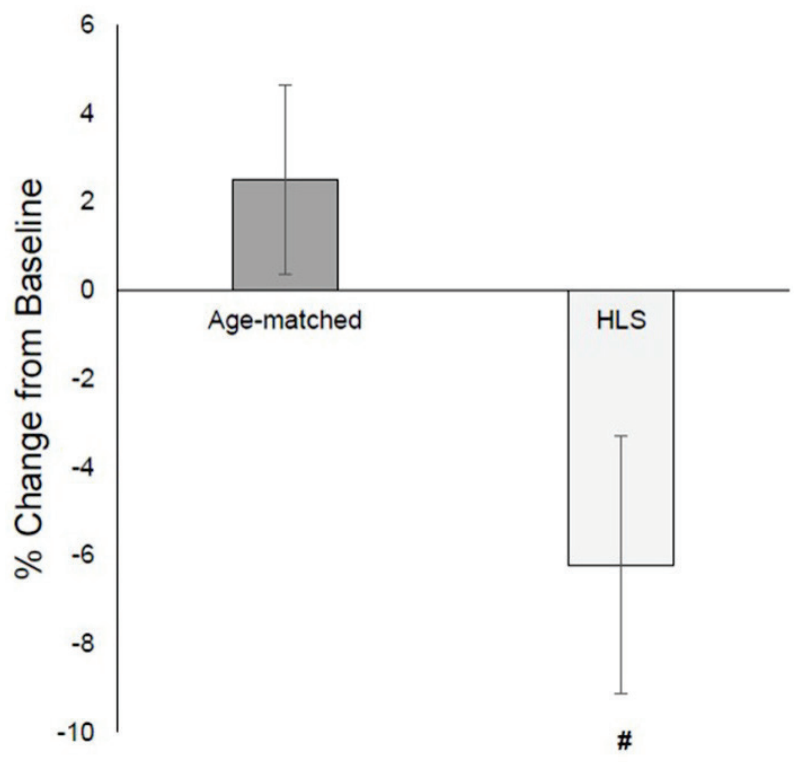

Fig. 1. Graph shows mean \pm SE values for the percent body weight change from baseline. The age-matched animals showed an increase in body weight by $2.5 \%$. On the other hand, HLS affected the percent body weight change with a decrease of $6.2 \%$ ( ${ }^{*} p<0.05$ vs. age-matched control).

\section{Soleus}

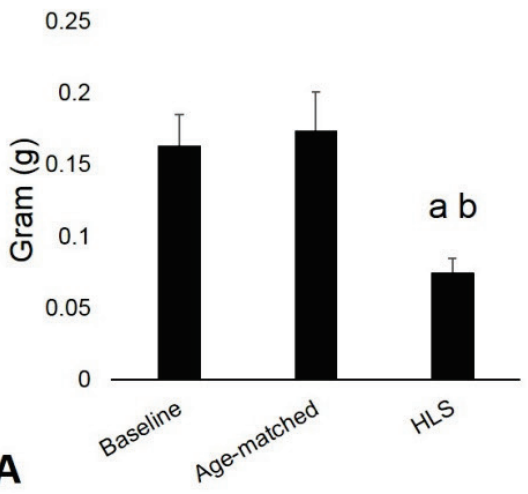

\section{Gastrocnemius}

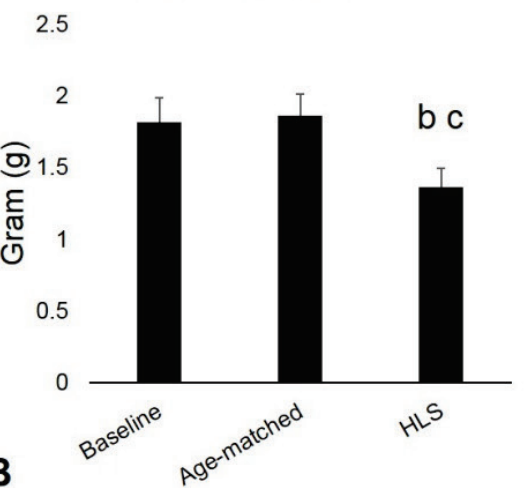

Quadriceps

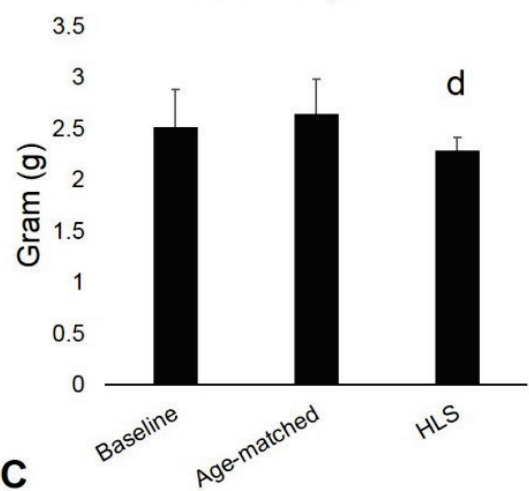

Fig. 2. Graph shows mean \pm SD values for the soleus $(\mathbf{A})$, gastrocnemius (B) and quadriceps (C) muscle wet weights. The muscle wet weights of the age-matched group were similar to the baseline. However, HLS significantly decreased the soleus wet weight compared to the baseline $\left({ }^{\mathbf{a}} p<0.001\right)$ and the age-matched $\left({ }^{\mathbf{b}} p<0.001\right)$ groups, and decreased the gastrocnemius wet weight compared to the baseline ( $\left.{ }^{\mathbf{c}} p<0.01\right)$ and the age-matched $\left({ }^{\mathbf{b}} p<0.001\right)$, as well as decreasing the quadriceps wet weight compared to age-matched (d $p<0.05)$. 
A

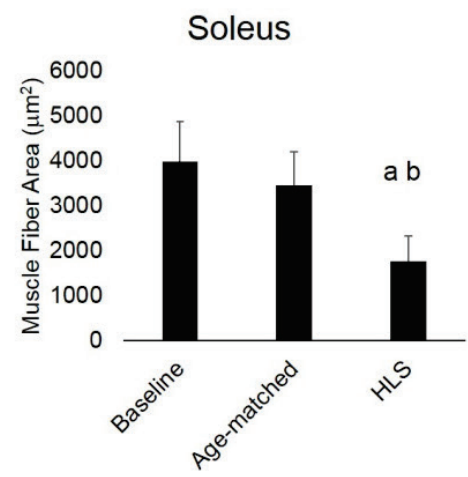

D

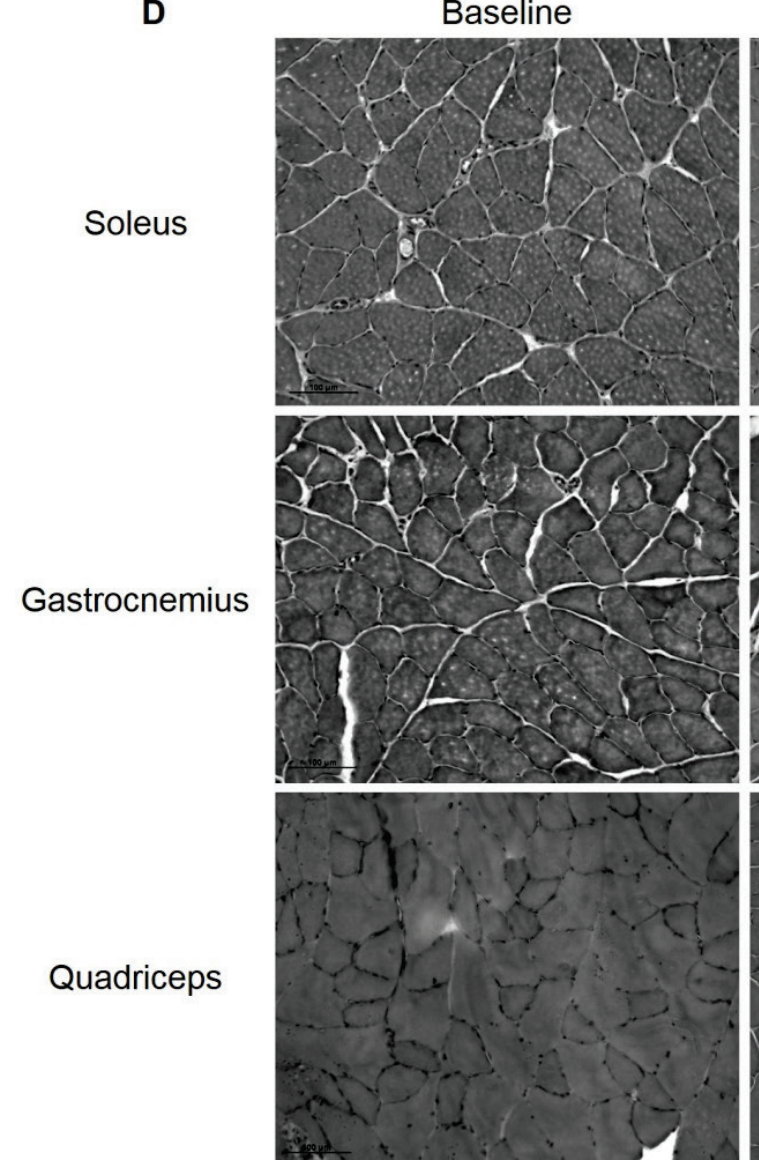

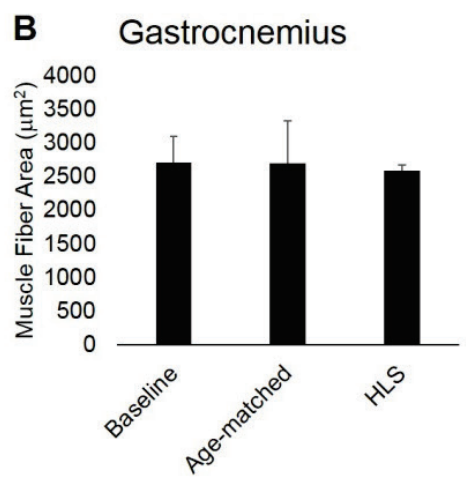

Age-matched
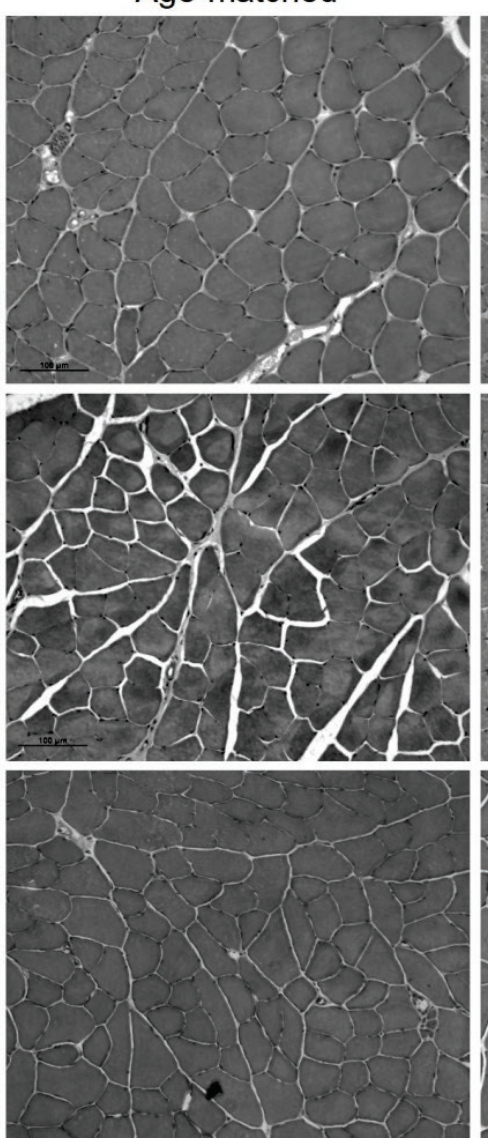

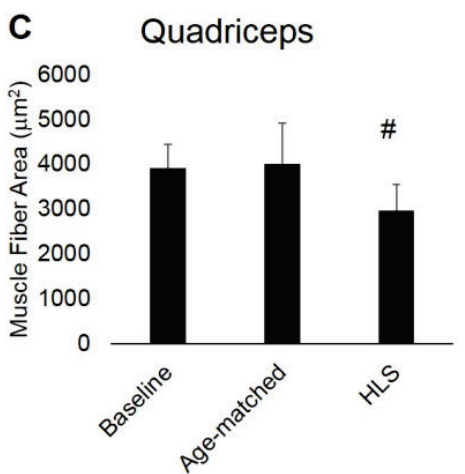

HLS
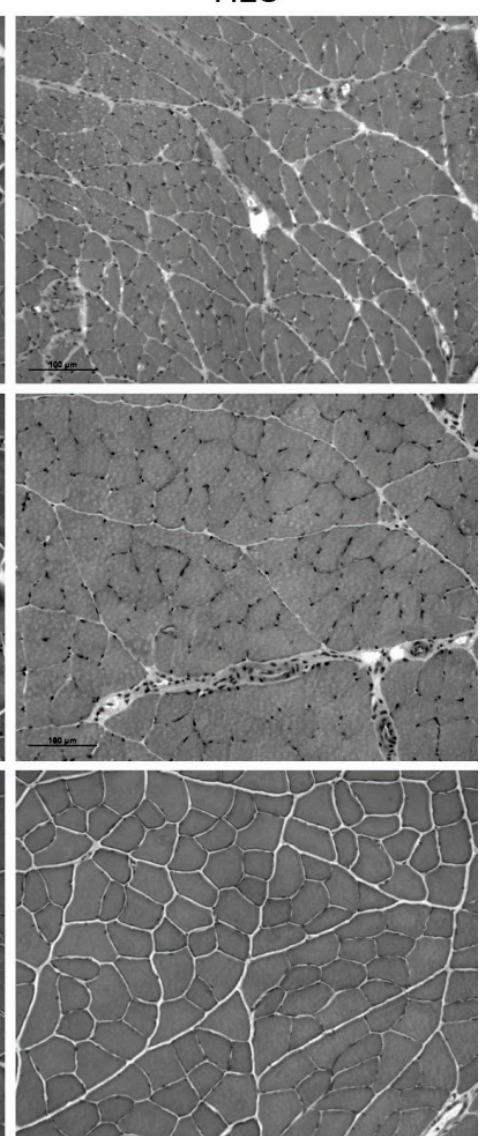

Fig. 3. Graph shows mean \pm SD values for the averaged cross-sectional soleus (A), gastrocnemius (B) and quadriceps (C) fiber area, along with representative histology images (D). HLS decreased the averaged cross-sectional muscle fiber area compared to baseline (for soleus) and age-matched control (for soleus and quadriceps). ${ }^{a} p<0.001$ vs. baseline; ${ }^{\mathbf{b}} p<0.01$ vs. age-matched; ${ }^{\#} p<0.05$ vs. age-matched.

DEMS at various loading frequencies induced change of cross-sectional muscle fiber area

As shown in Figure 4, the averaged crosssectional fiber area of the right soleus in the $50 \mathrm{~Hz}$ treatment group $\left(1,605 \pm 430 \mu \mathrm{m}^{2}\right)$ and $100 \mathrm{~Hz}$ treatment group $\left(855 \pm 75 \mu \mathrm{m}^{2}\right)$ were $18 \%(p>0.05)$ and $56 \%$ $(p<0.05)$ lower than the $1 \mathrm{~Hz}$ treatment group $\left(1,946 \pm 363 \mu \mathrm{m}^{2}\right)$, respectively. The right quadriceps in the $50 \mathrm{~Hz}$ treatment $\left(2,998 \pm 453 \mu \mathrm{m}^{2}\right)$ group was $8 \%$ larger than the $1 \mathrm{~Hz}$ treatment $\left(2,765 \pm 236 \mu \mathrm{m}^{2}\right)$ group $(p>0.05)$ and was $29 \%$ larger than the $100 \mathrm{~Hz}$ treatment $\left(2,329 \pm 148 \mu \mathrm{m}^{2}\right)$ group $(p<0.05)$. Although it was not statistically significant, the averaged cross-sectional gastrocnemius muscle fiber area showed a reduced value in the $100 \mathrm{~Hz}$ treatment $\left(1,953 \pm 711 \mu \mathrm{m}^{2}\right)$ compared to the $50 \mathrm{~Hz}\left(2,585 \pm 247 \mu \mathrm{m}^{2}\right)$ and the $1 \mathrm{~Hz}\left(2,583 \pm 91 \mu \mathrm{m}^{2}\right)$ treatment groups $(p>0.05)$. Normalization to the averaged HLS value showed similar trends. 


\section{Soleus}

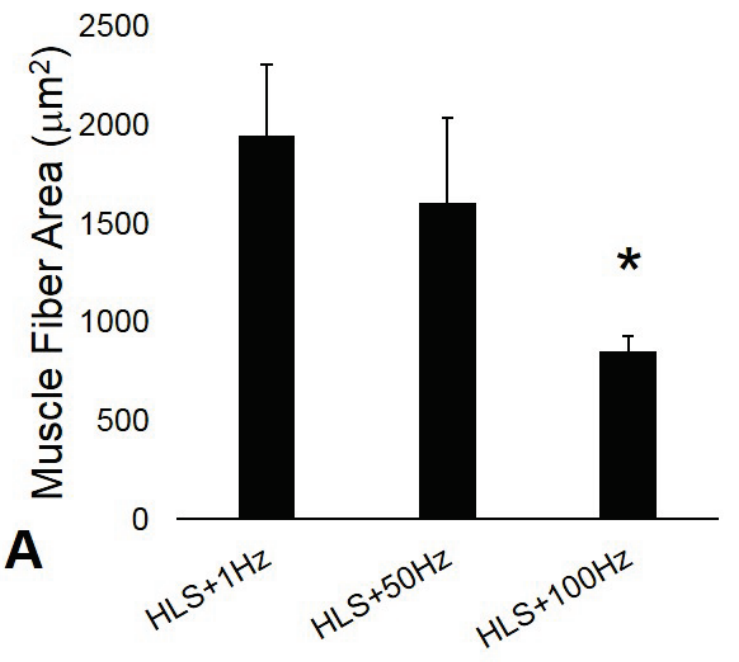

\section{Gastrocnemius}

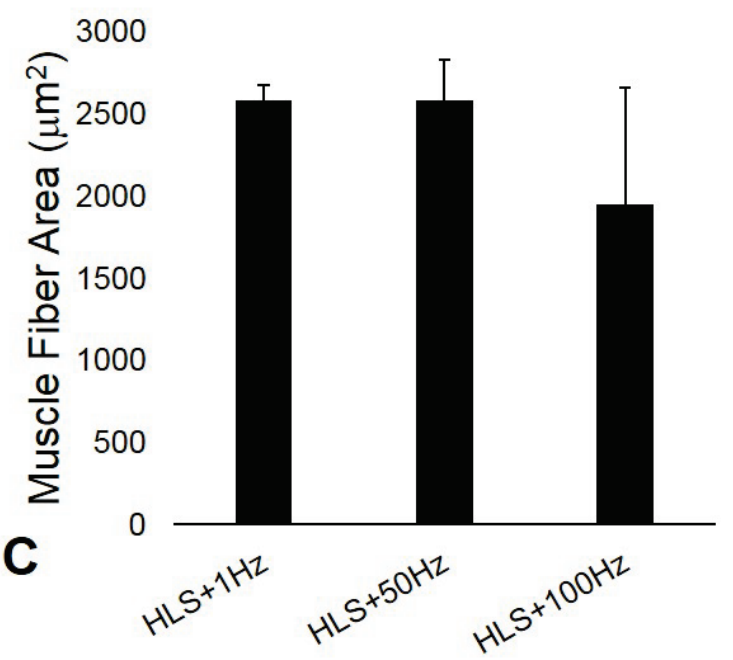

\section{Quadriceps}

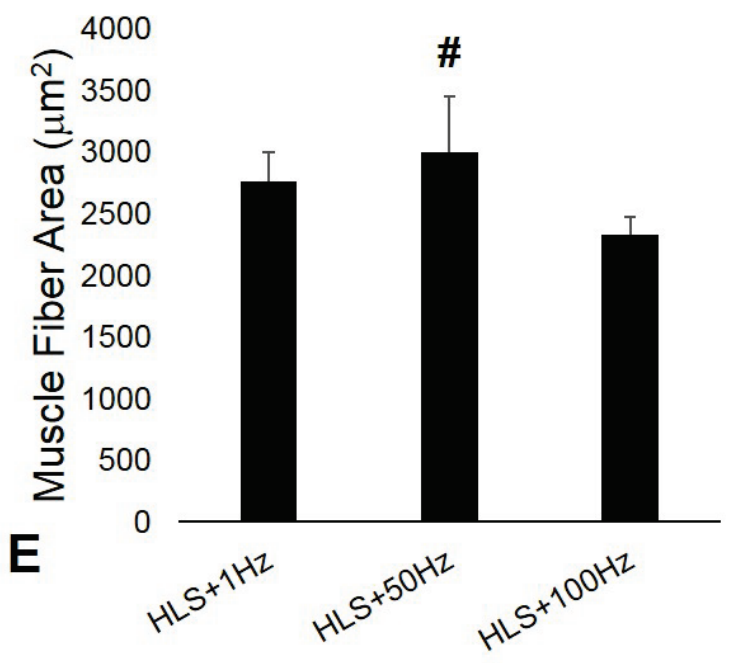

Soleus

1.4

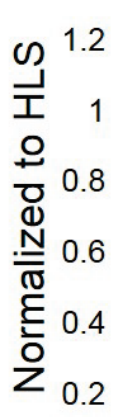

B
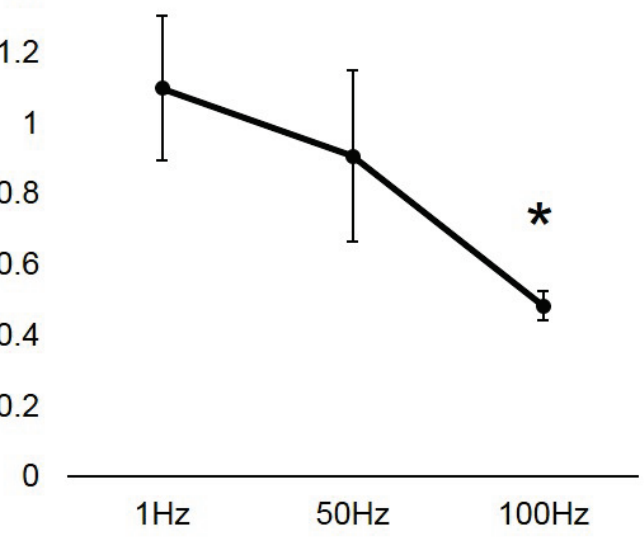

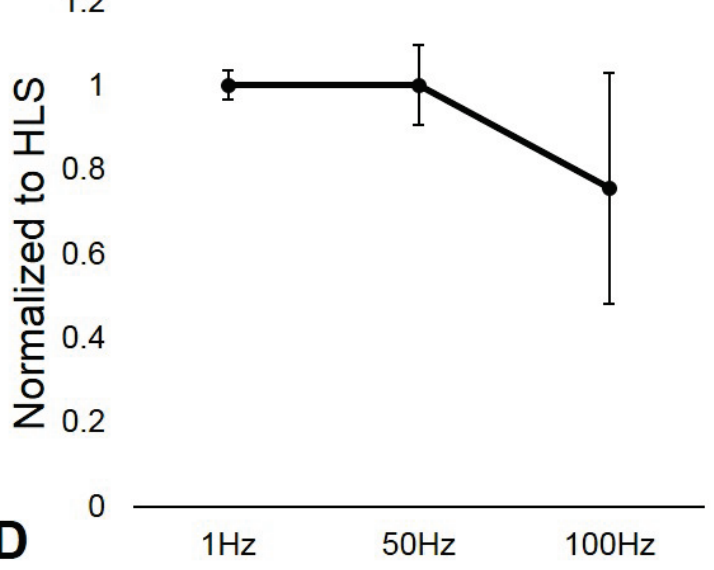

D

Gastrocnemius

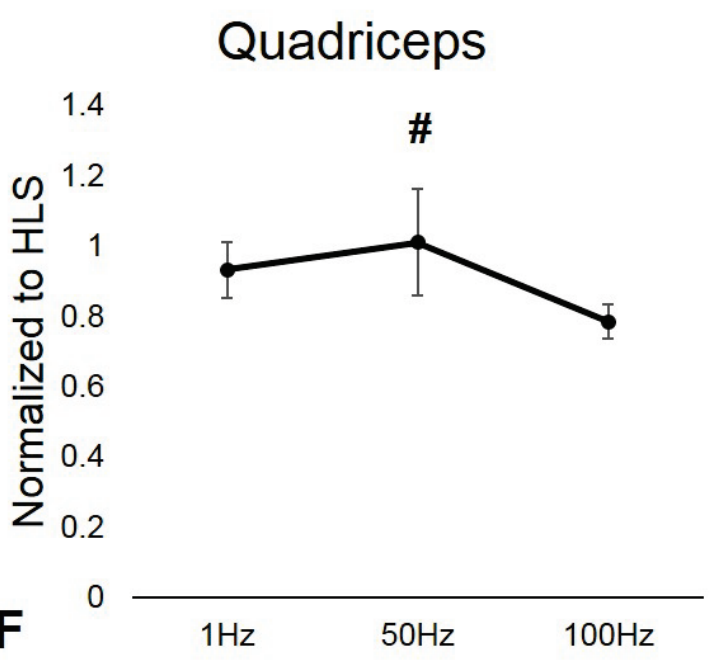

Fig. 4. Graph shows mean $\pm S D$ values for the averaged cross-sectional soleus, gastrocnemius and quadriceps fiber areas in response to DEMS at different loading frequencies (A, C and $\mathbf{E}$ ), as well as the corresponding normalization to the average HLS value (B, D and F). ${ }^{*} p<0.05$ vs. HLS $+1 \mathrm{~Hz} ;{ }^{*} p<0.05$ vs. HLS+100 Hz. 


\section{Soleus}

2000

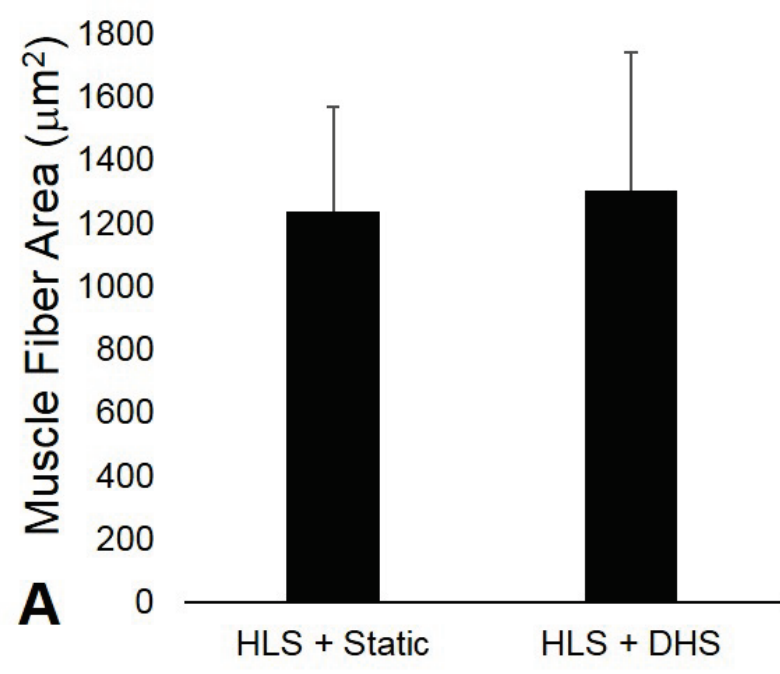

\section{Gastrocnemius}

4000

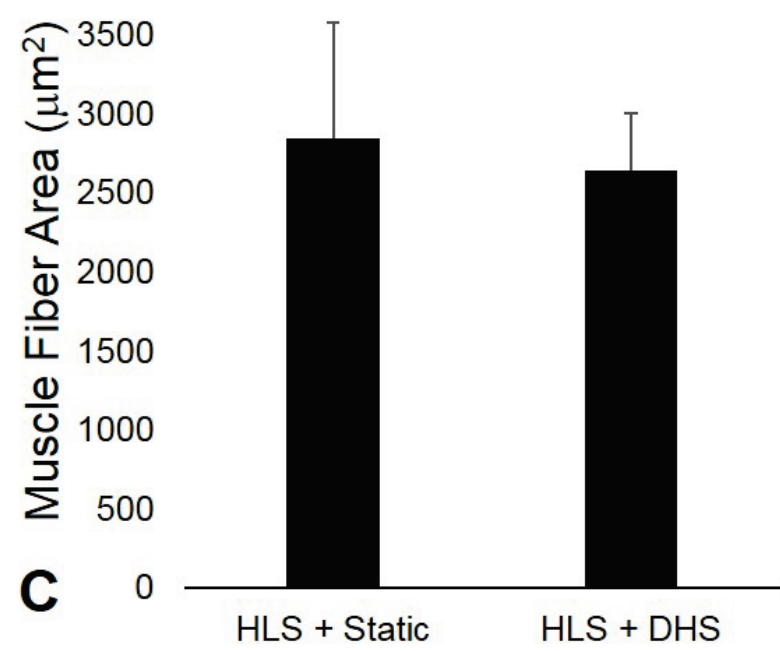

\section{Soleus}

\section{2}

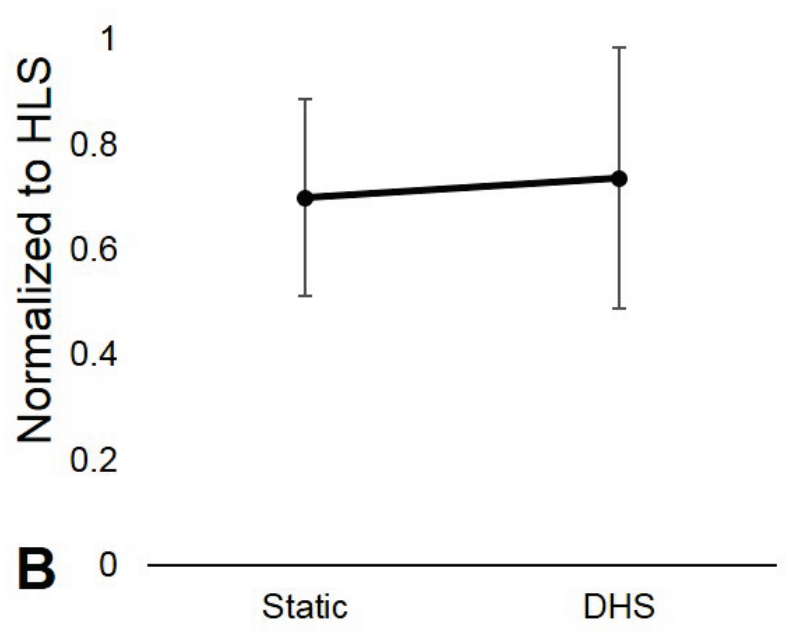

\section{Gastrocnemius}

\section{6}

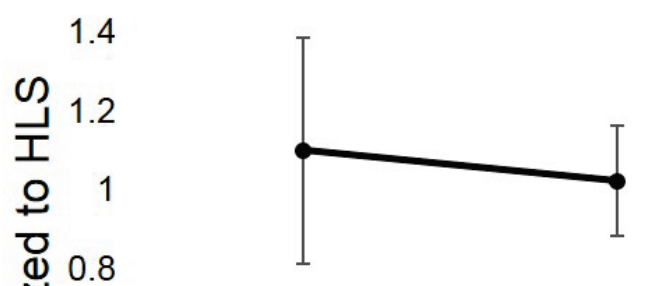

Fig. 5. Graph shows mean \pm SD values for the averaged cross-sectional soleus and gastrocnemius fiber area in response to static pressure and DHS loading ( $\mathbf{A}$ and $\mathbf{C}$ ), as well as the corresponding normalization to the average HLS value (B and $\mathbf{D})$. The averaged cross-sectional muscle fiber areas in the DHS were similar to the ones in the static group.

Unchanged cross-sectional muscle fiber area in response to $\mathrm{DHS}$

As shown in Figure 5, the averaged crosssectional fiber area of the right soleus in the DHS $\left(1,303 \pm 439 \mu \mathrm{m}^{2}\right)$ group was similar to the one in the static $\left(1,237 \pm 331 \mu \mathrm{m}^{2}\right)$ group $(p>0.05)$. Likewise, the difference between the averaged cross-sectional fiber areas of the right gastrocnemius muscles between the DHS $\left(2,643 \pm 363 \mu \mathrm{m}^{2}\right)$ group and the static $\left(2,843 \pm 739 \mu \mathrm{m}^{2}\right)$ group was statistically un-significant $(p>0.05)$. Normalization to the averaged HLS value showed similar trends.

\section{Discussion}

This study investigated the frequency effect of DEMS and the effect of the dynamic components derived from DHS on the muscle fiber morphological responses under disuse condition. HLS negatively affected the muscle wet weights and averaged cross-sectional fiber areas, more so in the soleus than in the gastrocnemius and quadriceps. Under such disuse condition, DEMS at $50 \mathrm{~Hz}$ 
had a significantly larger averaged cross-sectional fiber area compared to the $1 \mathrm{~Hz}$ or $100 \mathrm{~Hz}$ group in the quadriceps, and showed a similar trend in gastrocnemius. DEMS at $100 \mathrm{~Hz}$ showed a larger averaged crosssectional fiber area compared to the $1 \mathrm{~Hz}$ and $50 \mathrm{~Hz}$ stimulations in soleus. The quadriceps and gastrocnemius muscle are examples of fast-twitch muscles, which is highly responsive to contraction with abundant of type II muscle fibers (Zhou et al. 1995, Perez et al. 2002, Punkt et al. 2004). In contrast, the soleus is considered as a posture muscle (slow-twitch) with abundant of type I fibers (Punkt et al. 2004, Perez et al. 2002, Zhou et al. 1995). It has been reported that slow-twitch fibers are more sensitive to HLS than fast muscle fibers, and that disuse induces slow-to-fast transformation (Marsh et al. 1992, Oishi et al. 1998).

The present data confirmed that the effectiveness of DEMS is highly dependent on the stimulation frequency. Mechanical stimulations have been researched to alleviate muscle atrophy. Long duration $(10-24 \mathrm{~h})$ electrical stimulations at low frequency significantly prevented weight loss in soleus and tibialis anterior (Dupont Salter et al. 2003, Salmons and Henriksson 1981). However, others found that stimulation with higher frequency can maintain muscle mechanical properties, i.e. twitch and tetanic force, as well as reducing muscle weight loss and fiber CSA (Eerbeek et al. 1984, Gorza et al. 1988). Daily $30 \mathrm{~min}$ of electrical stimulation at $45 \mathrm{~Hz}-60 \mathrm{~Hz}$ was previously studied with healthy individuals (Perez et al. 2002). Since the subjects did not experience any disuse-related muscle loss, the stimulation served as an enhancement and showed an increased in averaged CSA by $14 \%$ and in the number of type II fibers. To our knowledge, this study was the first to examine the effect of such short-term duration (10 min) on disuse muscle. We have previously showed that DEMS, applied 10 min per day, can partially prevent trabecular bone loss in disused femur in a frequency dependent manner, where $50 \mathrm{~Hz}$ was found to be an optimized DEMS loading frequency for bone (Lam and Qin 2008). Correspondingly, the effect of DEMS on bone and muscle may ultimately give insights into the bone-muscle relationship.

The variation of stimulation frequencies within the DEMS regimen generated two types of isometric contraction at the quadriceps. DEMS at low frequency, such as $1 \mathrm{~Hz}$, produced isometric twitch, in which there is a rapid increase in muscle tension and then gradually return to normal. This induced twitch contractions, which failed to prevent muscle atrophy but was able to maintain maximum twitch tension (Yoshida et al. 2003). On the other hand, DEMS at mid to high frequency, such as $50 \mathrm{~Hz}$ or $100 \mathrm{~Hz}$, generated isometric tetanus, where a peak muscle tension is achieved by a summation effect (Gorza et al. 1988). However, from the characteristics of tissue material point of view, e.g. viscoelastic property in the tissues, both muscle and bone could quickly damp the loads at high frequencies (Qin and Lam 2009). Therefore, DEMS at $100 \mathrm{~Hz}$ was not observed to be as effective as the stimulation at $50 \mathrm{~Hz}$ in this study. For our studies, we concentrated in determining the morphological consequence of DEMS on the muscles under disuse condition. Previous studies by Gorza et al. measured the maximum tetanic tension of an innervated soleus to be $1.9 \mathrm{~N}$ and a denervated soleus to be $0.046 \mathrm{~N}$. The tension of the denervated muscle rose to $0.68 \mathrm{~N}$ with stimulation at $15 \mathrm{~Hz}$ and $1.1 \mathrm{~N}$ with stimulation at $100 \mathrm{~Hz}$ (Gorza et al. 1988). Similar results were found with HLS rats subjected to isometric exercise, the tetanic force of the soleus was increased by $30 \%$ (Hurst and Fitts 2003). The degree of protection with the stimulation was less when measured in a fast-twitch muscle, such as extensor digitorum longus. Nevertheless, the tension - frequency relation could potentially be one of the key elements in selecting the optimal stimulation frequency within the DEMS paradigm.

The $2 \mathrm{~Hz}$ dynamic component of DHS did not make a difference in muscle morphology compared to the static loading, under disuse condition. A consideration in the design of DEMS and DHS regimen is the elevation of fluid flow induced by each stimulation. DEMS and DHS have been shown to be able to raise intramuscular pressure (Hu et al. 2014, Hu et al. 2013a, Qin and Lam 2009). It is thought that rhythmical contractions increase venous outflow by compressing the arteries and veins, the vessels are refilled during relaxation (Valic et al. 2005). This concept of muscle pump is well appreciated and may be a potentially mechanism in the effects of DEMS. Treadmill exercise and 1 second of tetanic stimulation at $30 \mathrm{~Hz}$ both decreased arterial blood flow and increased venous blood flow at the onset of contraction (Valic et al. 2002). Blood flow then returned to normal level during rest period. In addition, blood expelled from the venous system increased as function of increased stimulation frequency (Hogan et al. 2003). Extensive researches also suggested other mechanisms, i.e. vasodilation, to explain the rapid increase in blood flow during the first few second of contraction (Buckwalter et al. 1998, Welsh and Segal 1997). 
Regardless of the possible mechanism, DEMS increased microvascular fluid perfusion is undeniable. Prolonged stimulation may enhance perfusion, augment oxygen and other metabolites delivery, and further protect the muscular tissues against the lack of functional activity. As a relatively passive stimulation, DHS's dynamic component at $2 \mathrm{~Hz}$ did not affect the muscle morphology under disuse, compared to the static application alone. Moreover, a limitation of this study was that the effect of DHS was only tested with a single frequency. While $2 \mathrm{~Hz}$ was found to be an optimized frequency for bone response (Hu et al. 2012, Hu et al. 2013a), it did not seem to induce muscle response. Further investigations should be done to optimize DHS's loading condition for muscle regeneration.

The quadriceps and gastrocnemius muscles are examples of fast-twitch muscles, which is highly responsive to contraction with abundant of type II muscle fibers. In contrast, the soleus is considered as a posture muscle (slow-twitch) with abundant of type I fibers (Zhou et al. 1995, Perez et al. 2002, Punkt et al. 2004). Studies on fast-twitch muscle groups, i.e., extensor digitorum longus (EDL), detected $\sim 95 \%$ of type II fibers via histochemical analysis and myosin heavy chain (MHC) II isoform via protein analysis (Jarvis et al. 1996, Punkt et al. 2004, Pette et al. 2002). Soleus expressed $\sim 85 \%$ of MHC II and $15 \%$ of MHC I (Desaphy et al. 2005). Mammalian skeletal muscle fibers display great adaptive ability via adjustments of their molecular, functional, and metabolic properties in response to altered functional demands (e.g. mechanical signals). Fiber type transitions occur in a sequential order, which results from the adaptive changes in the expression of myofibrillar and other protein isoforms. Alteration factors of the functional demand affect muscle fibers on their functional transitions in the direction of slow or fast (Pette 2002). It has been reported that slow-twitch fibers are more sensitive to HLS than fast muscle fibers, and that disuse induces slow-to-fast transformation (Marsh et al. 1992, Oishi et al. 1998) which continues during disuse (Staron et al. 1998). Soleus muscle containing different populations of slow type I fibers have various sensitivity to altered physiological conditions (Caiozzo et al. 1997). Suspension hypokinesia exclusively influences type I fibers in postural muscles (e.g. SOL), and leads to arrest of muscle developmental conversion of type II to type I fibers (Asmussen and Soukup 1991). Marked increases of rat $\mathrm{MHCIId} / \mathrm{x}$ and MHCIIb isoforms as well as pronounced decreases in slow MHCI were induced by phasic high-frequency stimulation exceeded by far those of denervation, leading to marked increases of these two isoforms, as well as to pronounced decreases in slow MHCI (Hamalainen and Pette 1996). A graded suppressive effect of thyroid hormone on the expression of MHC isoforms was in the order of MHCIId less than MHCIIa less than MHCI, which was partially counteracted by elevated neuromuscular activity and showed a similar relationship for the slow MHCI and the slow MLC isoforms (Kirschbaum et al. 1990). One limitation of this current study is that we did not collect and specifically preserve the muscle samples for muscle fiber type analysis. As a preliminary study, we would like to perform initial assessments and comparison on the mitigation effects of DEMS and DHS on muscle atrophy under disuse condition. We will expand our research scope into muscle fiber type responses and systemic assessments (e.g. blood circulation) to further compare the effects and mechanisms of DEMS and DHS.

In conclusion, $10 \mathrm{~min}$ of daily DEMS to the quadriceps at $50 \mathrm{~Hz}$ showed an increase averaged crosssectional quadriceps fiber area, compared to $1 \mathrm{~Hz}$ and $100 \mathrm{~Hz}$. The variation of stimulation frequency has impacted on the morphological change of the muscle fibers under disuse condition. On the other hand, no difference in muscle morphology was found between the static and $2 \mathrm{~Hz}$ DHS stimulations. Although the mechanism between stimulation and tissue adaptation remain unclear, these studies demonstrated the importance in considering the type of stimulation that is being applied and the changes in fluid perfusion that is being generated by such stimulation. Future investigation may target the duration of the stimulations and supplement with the appropriate stimulation frequency within the DEMS and DHS regimens. It is aimed to optimize the DHS loading conditions (e.g. frequency and magnitude) effective on muscle and to determine the downstream mechanisms.

\section{Conflict of Interest}

There is no conflict of interest.

\section{Acknowledgements}

The authors are thankful for the kind support from National Institute of Health (R01 AR52379 and AR61821, YXQ), US Army Medical Research and Materiel Command, and National Space Biomedical Research Institute through NASA Cooperative Agreement NCC 9-58 (YXQ). 


\section{References}

ALLEN MR, BLOOMFIELD SA: Hindlimb unloading has a greater effect on cortical compared with cancellous bone in mature female rats. J Appl Physiol 94: 642-650, 2003.

ASMUSSEN G, SOUKUP T: Arrest of developmental conversion of type II to type I fibres after suspension hypokinesia. Histochem J 23: 312-322, 1991.

BALDI JC, JACKSON RD, MORAILLE R, MYSIW WJ: Muscle atrophy is prevented in patients with acute spinal cord injury using functional electrical stimulation. Spinal Cord 36: 463-469, 1998.

BONEWALD LF, KIEL DP, CLEMENS TL, ESSERK, ORWOLL ES, O'KEEFE RJ, FIELDING RA: Forum on bone and skeletal muscle interactions: summary of the proceedings of an ASBMR workshop. J Bone Miner Res 28 : 1857-1865, 2013.

BOONYAROM O, INUI K: Atrophy and hypertrophy of skeletal muscles: structural and functional aspects. Acta Physiol (Oxf) 188: 77-89, 2006.

BUCKWALTER JB, RUBLE SB, MUELLER PJ, CLIFFORD PS: Skeletal muscle vasodilation at the onset of exercise. J Appl Physiol 85: 1649-1654, 1998.

CAIOZZO VJ, BAKER MJ, MCCUE SA, BALDWIN KM: Single-fiber and whole muscle analyses of MHC isoform plasticity: interaction between T3 and unloading. Am J Physiol 273: C944-C952, 1997.

CASTRO MJ, APPLE DF JR, STARON RS, CAMPOS GE, DUDLEY GA: Influence of complete spinal cord injury on skeletal muscle within 6 mo of injury. J Appl Physiol 86: 350-358, 1999.

CIANFEROTTI L, BRANDI ML: Muscle-bone interactions: basic and clinical aspects. Endocrine 45: 165-177, 2014.

DESAPHY JF, PIERNO S, LIANTONIO A, DE LUCA A, DIDONNA MP, FRIGERI A, NICCHIA GP, SVELTO M, CAMERINO C, ZALLONE A, CAMERINO DC: Recovery of the soleus muscle after short- and long-term disuse induced by hindlimb unloading: effects on the electrical properties and myosin heavy chain profile. Neurobiol Dis 18: 356-365, 2005.

DUPONT SALTER AC, RICHMOND FJ, LOEB GE: Prevention of muscle disuse atrophy by low-frequency electrical stimulation in rats. IEEE Trans Neural Syst Rehabil Eng 11: 218-226, 2003.

EDGERTON VR, ZHOU MY, OHIRA Y, KLITGAARD H, JIANG B, BELL G, HARRIS B, SALTIN B, GOLLNICK PD, ROY RR, DAY MK, GREENISEN M: Human fiber size and enzymatic properties after 5 and 11 days of spaceflight. J Appl Physiol 78: 1733-1739, 1995.

EERBEEK O, KERNELL D, VERHEY BA: Effects of fast and slow patterns of tonic long-term stimulation on contractile properties of fast muscle in the cat. J Physiol 352: 73-90, 1984.

FISHER JS, HASSER EM, BROWN M: Effects of ovariectomy and hindlimb unloading on skeletal muscle. $J$ Appl Physiol 85: 1316-1321, 1998.

GERRITS HL, HOPMAN MT, SARGEANT AJ, JONES DA, DE HAAN A: Effects of training on contractile properties of paralyzed quadriceps muscle. Muscle Nerve 25: 559-567, 2002.

GORZA L, GUNDERSEN K, LOMO T, SCHIAFFINO S, WESTGAARD RH: Slow-to-fast transformation of denervated soleus muscles by chronic high-frequency stimulation in the rat. J Physiol 402: 627-649, 1988.

GREGORY CM, VANDENBORNE K, CASTRO MJ, DUDLEY GA: Human and rat skeletal muscle adaptations to spinal cord injury. Can J Appl Physiol 28: 491-500, 2003.

HAMALAINEN N, PETTE D: Slow-to-fast transitions in myosin expression of rat soleus muscle by phasic high-frequency stimulation. FEBS Lett 399: 220-222, 1996.

HAUSCHKA EO, ROY RR, EDGERTON VR: Periodic weight support effects on rat soleus fibers after hindlimb suspension. J Appl Physiol 65: 1231-1237, 1988.

HOGAN MC, GRASSI B, SAMAJA M, STARY CM, GLADDEN LB: Effect of contraction frequency on the contractile and noncontractile phases of muscle venous blood flow. J Appl Physiol 95: 1139-1144, 2003.

HU M, QIN YX: Dynamic fluid flow stimulation on cortical bone and alterations of the gene expressions of osteogenic growth factors and transcription factors in a rat functional disuse model. Arch Biochem Biophys 545: 154-161, 2014. 
HU M, CHENG J, BETHEL N, SERRA-HSU F, FERRERI S, LIN L, QIN YX: Interrelation between external oscillatory muscle coupling amplitude and in vivo intramedullary pressure related bone adaptation. Bone 66 : 178-181, 2014.

HU M, CHENG J, QIN YX: Dynamic hydraulic flow stimulation on mitigation of trabecular bone loss in a rat functional disuse model. Bone 51: 819-825, 2012.

HU M, SERRA-HSU F, BETHEL N, LIN L, FERRERI S, CHENG J, QIN YX: Dynamic hydraulic fluid stimulation regulated intramedullary pressure. Bone 57: 137-141, $2013 \mathrm{a}$.

HU M, YEH R, LIEN M, TEERATANANON M, AGARWAL K, QIN Y: Dynamic fluid flow mechanical stimulation modulates bone marrow mesenchymal stem cells. Bone Research 1, 98-104, 2013b.

HURST JE, FITTS RH: Hindlimb unloading-induced muscle atrophy and loss of function: protective effect of isometric exercise. J Appl Physiol 95: 1405-1417, 2003.

JARVIS JC, SUTHERLAND H, MAYNE CN, GILROY SJ, SALMONS S: Induction of a fast-oxidative phenotype by chronic muscle stimulation: mechanical and biochemical studies. Am J Physiol 270: C306-C312, 1996.

KERNELL D, EERBEEK O, VERHEY BA, DONSELAAR Y: Effects of physiological amounts of high- and low-rate chronic stimulation on fast-twitch muscle of the cat hindlimb. I. Speed- and force-related properties. J Neurophysiol 58: 598-613, 1987.

KIRSCHBAUM BJ, KUCHER HB, TERMIN A, KELLY AM, PETTE D: Antagonistic effects of chronic low frequency stimulation and thyroid hormone on myosin expression in rat fast-twitch muscle. $J$ Biol Chem 265 : 13974-13980, 1990.

KYPAROS A, FEEBACK DL, LAYNE CS, MARTINEZ DA, CLARKE MS: Mechanical stimulation of the plantar foot surface attenuates soleus muscle atrophy induced by hindlimb unloading in rats. J Appl Physiol 99: 739-746, 2005.

LAM H, HU M, QIN YX: Alteration of contraction-to-rest ratio to optimize trabecular bone adaptation induced by dynamic muscle stimulation. Bone 48: 399-405, 2011.

LAM H, QIN YX: The effects of frequency-dependent dynamic muscle stimulation on inhibition of trabecular bone loss in a disuse model. Bone 43: 1093-1100, 2008.

LEBLANC A, LIN C, SHACKELFORD L, SINITSYN V, EVANS H, BELICHENKO O, SCHENKMAN B, KOZLOVSKAYA I, OGANOV V, BAKULIN A, ET AL.: Muscle volume, MRI relaxation times (T2), and body composition after spaceflight. J Appl Physiol 89: 2158-2164, 2000.

MABUCHI K, SZVETKO D, PINTER K, SRETER FA: Type IIB to IIA fiber transformation in intermittently stimulated rabbit muscles. Am J Physiol 242: C373-C381, 1982.

MARSH DR, CAMPBELL CB, SPRIET LL: Effect of hindlimb unweighting on anaerobic metabolism in rat skeletal muscle. J Appl Physiol 72: 1304-1310, 1992.

MCCLUNG JM, DAVIS JM, WILSON MA, GOLDSMITH EC, CARSON JA: Estrogen status and skeletal muscle recovery from disuse atrophy. J Appl Physiol 100: 2012-2023, 2006.

MOREY-HOLTON ER, GLOBUS RK: Hindlimb unloading of growing rats: a model for predicting skeletal changes during space flight. Bone 22: 83S-88S, 1998.

MOREY-HOLTON ER, GLOBUS RK: Hindlimb unloading rodent model: technical aspects. J Appl Physiol 92: 1367-1377, 2002.

NARICI M, KAYSER B, BARATTINI P, CERRETELLI P: Effects of 17-day spaceflight on electrically evoked torque and cross-sectional area of the human triceps surae. Eur J Appl Physiol 90: 275-282, 2003.

OISHI Y, ISHIHARA A, YAMAMOTO H, MIYAMOTO E: Hindlimb suspension induces the expression of multiple myosin heavy chain isoforms in single fibres of the rat soleus muscle. Acta Physiol Scand 162: 127-134, 1998.

PEREZ M, LUCIA A, RIVERO JL, SERRANO AL, CALBET JA, DELGADO MA, CHICHARRO JL: Effects of transcutaneous short-term electrical stimulation on $\mathrm{M}$. vastus lateralis characteristics of healthy young men. Pflugers Arch 443: 866-874, 2002.

PETTE D: The adaptive potential of skeletal muscle fibers. Can J Appl Physiol 27: 423-448, 2002.

PETTE D, SKETELJ J, SKORJANC D, LEISNER E, TRAUB I, BAJROVIC F: Partial fast-to-slow conversion of regenerating rat fast-twitch muscle by chronic low-frequency stimulation. J Muscle Res Cell Motil 23: 215-221, 2002. 
PUNKT K, NAUPERT A, ASMUSSEN G: Differentiation of rat skeletal muscle fibres during development and ageing. Acta Histochem 106: 145-154, 2004.

QIN YX, LAM H: Intramedullary pressure and matrix strain induced by oscillatory skeletal muscle stimulation and its potential in adaptation. $J$ Biomech 42: 140-145, 2009.

SALMONS S, HENRIKSSON J: The adaptive response of skeletal muscle to increased use. Muscle Nerve 4: 94-105, 1981.

SKOLD C, LONN L, HARMS-RINGDAHL K, HULTLING C, LEVI R, NASH M, SEIGER A: Effects of functional electrical stimulation training for six months on body composition and spasticity in motor complete tetraplegic spinal cord-injured individuals. J Rehabil Med 34: 25-32, 2002.

SPUNGEN AM, ADKINS RH, STEWART CA, WANG J, PIERSON RN JR, WATERS RL, BAUMAN WA: Factors influencing body composition in persons with spinal cord injury: a cross-sectional study. J Appl Physiol 95 : 2398-2407, 2003.

STARON RS, KRAEMER WJ, HIKIDA RS, REED DW, MURRAY JD, CAMPOS GE, GORDON SE: Comparison of soleus muscles from rats exposed to microgravity for 10 versus 14 days. Histochem Cell Biol 110: 73-80, 1998.

TRAPPE SW, TRAPPE TA, LEE GA, WIDRICK JJ, COSTILL DL, FITTS RH: Comparison of a space shuttle flight (STS-78) and bed rest on human muscle function. J Appl Physiol 91: 57-64, 2001.

VALIC Z, BUCKWALTER JB, CLIFFORD PS: Muscle blood flow response to contraction: influence of venous pressure. J Appl Physiol 98: 72-76, 2005.

VALIC Z, NAIK JS, RUBLE SB, BUCKWALTER JB, CLIFFORD PS: Elevation in resting blood flow attenuates exercise hyperemia. J Appl Physiol 93: 134-140, 2002.

WELSH DG, SEGAL SS: Coactivation of resistance vessels and muscle fibers with acetylcholine release from motor nerves. Am J Physiol 273: H156-H163, 1997.

YOSHIDA N, SAIRYO K, SASA T, FUKUNAGA M, KOGA K, IKATA T, YASUI N: Electrical stimulation prevents deterioration of the oxidative capacity of disuse-atrophied muscles in rats. Aviat Space Environ Med 74: 207-211, 2003.

ZEHNDER Y, LUTHI M, MICHEL D, KNECHT H, PERRELET R, NETO I, KRAENZLIN M, ZACH G, LIPPUNER $\mathrm{K}$ : Long-term changes in bone metabolism, bone mineral density, quantitative ultrasound parameters, and fracture incidence after spinal cord injury: a cross-sectional observational study in 100 paraplegic men. Osteoporos Int 15: 180-189, 2004.

ZHOU MY, KLITGAARD H, SALTIN B, ROY RR, EDGERTON VR, GOLLNICK PD: Myosin heavy chain isoforms of human muscle after short-term spaceflight. J Appl Physiol 78: 1740-1744, 1995. 